\title{
Erratum to: Observation of yttrium oxide nanoparticles in cabbage (Brassica oleracea) through dual energy K-edge subtraction imaging
}

Yunyun Chen ${ }^{1}$, Carlos Sanchez ${ }^{2}$, Yuan Yue ${ }^{1}$, Mauricio de Almeida ${ }^{3}$, Jorge M. González ${ }^{3}$, Dilworth Y. Parkinson ${ }^{4}$ and Hong Liang ${ }^{1,2^{*}}$

\section{Erratum to: J Nanobiotechnol (2016) 14:23 DOI 10.1186/s12951-016-0175-z}

After publication of this article [1], we received support to pay the article processing charge. Part of the open access publishing fee for this manuscript has been provided by the Texas A\&M University Online Access to Knowledge Fund (OAKFund), supported by the University Libraries and the Office of the Vice President for Research.

\section{Author details}

${ }^{1}$ Materials Science and Engineering, Texas A\&M University, College Station, TX 77843-3123, USA. ${ }^{2}$ Mechanical Engineering, Texas A\&M University, College Station, TX 77843-3123, USA. ${ }^{3}$ Department of Plant Science, California State University, Fresno, CA 93740, USA. ${ }^{4}$ Advanced Light Source, Lawrence Berkeley National Laboratory, Berkeley, CA 94720, USA.

The online version of the original article can be found under doi:10.1186/s12951-016-0175-z.

Received: 12 April 2016 Accepted: 12 April 2016

Published online: 20 April 2016

\section{Reference}

1. Chen Y, et al. Observation of yttrium oxide nanoparticles in cabbage

(Brassica oleracea) through dual energy K-edge subtraction imaging. J

Nanobiotechnol. 2016;14:23.

\footnotetext{
*Correspondence: hliang@tamu.edu

1 Materials Science and Engineering, Texas A\&M University, College Station, TX 77843-3123, USA

Full list of author information is available at the end of the article
} 